\title{
Invited commentary on: Fear reduction
}

\section{by psychotherapies ${ }^{\dagger}$}

\section{TREATMENT OF ANXIETY}

A quarter of a century ago I commented upon a review of psychotherapeutic practice:

"In his review of psychotherapeutic practice today, Marks has pointed out that there is a decreasing tendency to make dogmatic assertions of universal applicability of one or other form of psychotherapy to all neurotic states. There is an increasing readiness among psychotherapists to recognise that genuine psychotherapeutic potential may be found in a variety of techniques with widely different assumptions about the nature of neurosis ... It is becoming unrealistic to expect that all neurotic problems will be solved by one technique alone (Snaith, 1974)".

The survey published here purports to narrow the field of neurotic disorder to disproportionate anxiety (or fear) and excludes those forms of therapy generally subsumed under the generic term 'psychodynamic'. However, even within this narrower confine the conclusion seems to be much the same, that is, that all elements (or at least those mentioned in the survey) have therapeutic potential and that any assertion of superiority of one approach over another is unwarranted. Indeed, the survey concludes that an underlying element is likely to be confidence: I would expand this and say that effective therapy will depend upon the patient having confidence in the competence of the therapist, in the approach to treatment proposed and finally in ability to master the distress of the disorder. In their 'map' for fear reduction the authors, true to behavioural stance, still place exposure first ('the highway') and leave stress mastery through meditation as an 'indistinct trail', a sort of also-ran perhaps to be thrown in as an adjunct possibly if other elements have failed to produce sufficient effect. It is all very well to highlight the splendid effect of exposure but, as said, a patient must have confidence to engage in

†See editorial pp. 507-5II, this issue. the therapy and advocacy of confrontation with the source of the anxiety takes no account of the proportion of patients who would decline to participate in the method proposed, which is likely to considerably exceed that in other approaches. It was indeed the experience that so many patients rejected treatment by exposure to the source of their anxiety, as well as difficulty in replicating such scenarios in the clinic, that led me to espouse the approach through meditational practice, varieties of which (including my own development of a technique I have called anxiety control training (ACT)) I have recently reviewed (Snaith, 1998). The essence of this approach is the establishment of self-control through an easily taught coping method and it is a method which I have found to be very acceptable by those to whom it is offered. In the procedure exposure to anxiety may be included, but that is at the discretion of the patient who may find that he or she is making sufficient improvement without such imaginal exposure. It has been insufficiently researched but, since Marks \& Dar omit mention of the method, I will outline the two published articles based upon it. In the first of these (Constantopoulos et al, 1982) we were interested to try to determine whether or not the addition of anxiety exposure in the setting of ACT was necessary for improvement; the answer was that it was not and patients made similar improvement if they were simply encouraged, in the setting of ACT practice, to rehearse self-coping statements along the lines advocated by Meichenbaum in his technique of stress inoculation training. In the second study (Snaith et al, 1992) we were interested to determine whether, having been shown the technique, patients, left to themselves, could maintain the improvement over a long period or whether continued contact with the therapist was necessary to maintain improvement; this study had the merits of random allocation, a sufficiently large sample, fully independent and blind assessment and regular assessments over a nine-month period by instruments sensitive to change. No patient declined the technique after explanation of the principles of ACT. We were pleased to report that fall-out was very low, improvement was maintained and, moreover, that this increased throughout the period of follow-up and that continuing contact with the therapist was unnecessary: the conclusion was that a self-mastery device had been acquired through which self-confidence continued to grow (Snaith et al, 1992). These findings are surely worth more attention and research than a dismissal into a side-stream approach to anxiety management.

My next comment upon the review is on the need for careful attention to the psychopathology of the disorders treated. For instance the authors include obsessivecompulsive disorder (OCD) as form of anxiety disorder; in this they follow the DSM-IV (American Psychiatric Association, 1994) but the ICD-10 (World Health Organization, 1992) does not regard the nature of OCD as being based upon anxiety; it frequently occurs in the setting of depressive illness and indeed many studies confirm that the disorder is likely to respond to antidepressant medication. I request that the overall title of 'generalised anxiety disorder', as used by the reviewers, requires better attention as to the psychopathological basis of the disorder in any particular case; in many cases it is a manifestation of a long-standing trait of proneness to anxiety, in others it is based upon ongoing severe stress or conflict but in others again it is a manifestation of an underlying affective disorder of a type likely to respond to antidepressant medication or, as is the case with recurrent depressive illness, to improve spontaneously. It is worth pointing out that the term 'depression' is likewise overinclusive of a variety of psychopathological states; in an attempt to wrestle with this particular problem we followed the view of Klein (1974) that the manifestation of depressive disorder most likely to predict a response to antidepressant medication is the presence of marked lowering of ability to experience pleasure, the totality of which is termed anhedonia. Therefore, in the devising of a simple selfassessment questionnaire, the Hospital Anxiety and Depression Scale (Zigmond \& Snaith, 1983), we took care to separate the constructs of generalised anxiety and depression and to base the latter largely 
(although not entirely) on diminished hedonic capacity. This has served us well as a preliminary screening device to help determine which patients presenting with generalised anxiety should first be offered treatment with antidepressant medication.

It must be disappointing for Marks \& Dar to have to indicate that knowledge about anxiety management remains in an anarchic state. They give some thoughts upon the directions of future research. I hope that this commentary on their article may add to their thoughts.
American Psychiatric Association (1994) Diagnostic and Statistical Manual of Mental Disorders (4th edn) (DSM-IV). Washington, DC: APA

Constantopoulos, A., Snaith, R. P. \& Jardine, Y. (1982) Self-control psychotherapy with and without exposure to anxiety. In Learning Theory Approaches To Psychiatry, pp. III-II5. New York \& Chichester: John Wiley and Sons.

Klein, D. F. (1974) Endogenomorphic depression. Archives of General Psychiatry, 3I, 447-454.

Snaith, R. P. (1974) A method of psychotherapy based on relaxation techniques. British Journal of Psychiatry, $\mathbf{1 2 4}$ 473-48I.

- (1998) Meditation and psychotherapy. British Journal of Psychiatry, 173, 193-195.
—, Owens, D. \& Kennedy, E. (1992) An outcome study of a brief anxiety management programme: Anxiety Control Training. Irish Journal of Psychological Medicine, 9, III-II4.

World Health Organization (1992) Tenth Revision of the International Classification of Diseases and Related Health Problems (ICD-10). Geneva: WHO.

Zigmond, A. S. \& Snaith, R. P. (1983) The Hospital Anxiety and Depression Scale. Acta Psychiatrica Scandinavica, 67, 36I-370.

Philip Snaith 30 Gledhow Wood Road, Leeds LS8 4BZ. e-mail: rpsnaith@pipemedia.co.uk 